\title{
IS6110 Restriction Fragment Length Polymorphism of Mycobacterium tuberculosis Isolated from Patients with Pulmonary Tuberculosis in Campinas, Brazil. Evidence of Intercontinental Distribution of Strains
}

\author{
Ana Lucia Roscani Calusni, Gláucia Nogueira Roscani, Maria Cecília Barisson Villares, \\ Hanna Soini*, Edward A Graviss**, Marcelo de Carvalho Ramos/ ${ }^{+}$
}

\begin{abstract}
Laboratório de Patogênese Bacteriana, Departamento de Clínica Médica, Faculdade de Ciências Médicas, Universidade Estadual de Campinas, Cidade Universitária Zeferino Vaz, 13083-970 Campinas, SP, Brasil *Mycobacterial Reference Laboratory, National Public Health Institute, Turku, Finland **Houston Tuberculosis Initiative, Baylor College of Medicine, Houston, Texas, US
\end{abstract}

Tuberculosis (TB) is a major concern in developing countries. In Brazil, few genotyping studies have been conducted to verify the number of IS6110 copies present in local prevalent strains of Mycobacterium tuberculosis, the distribution and clustering of strains. IS6110 DNA fingerprinting was performed on a sample of M. tuberculosis isolates from patients with AFB smear-positive pulmonary TB, at a hospital in Brazil. The IS6110 profiles were analyzed and compared to a M. tuberculosis database of the Houston Tuberculosis Initiative, Houston, US. Seventysix fingerprints were obtained from 98 patients. All M. tuberculosis strains had an IS6110 copy number between 5-21 allowing for differentiation of the isolates. Human immunodeficiency virus infection was confirmed in nearly half the patients of whom data was available. Fifty-eight strains had unique patterns, while 17 strains were grouped in 7 clusters (2 to 6 strains). When compared to the HTI database, 6 strains matched isolates from El Paso, Ciudad de Juarez, Houston, and New York. Recently acquired infections were documented in 19\% of cases. The community transmission of infection is intense, since some clustered strains were recovered during the four-year study period. The intercontinental dissemination of $\mathrm{M}$. tuberculosis strains is suspected by demonstration of identical fingerprints in a distant country.

Key words: tuberculosis - Mycobacterium tuberculosis - IS6110 restriction fragment length polymorphism

Tuberculosis (TB) remains one of the most important infectious causes of death in the world, both in industrialized and developing countries (Soini et al. 2001). The incidence and morbidity from TB have been greatly exacerbated by the human immunodeficiency virus (HIV) pandemic (Hopewell 1989). Active disease produced by Mycobacterium tuberculosis, develops either following a recently acquired infection (primary) or from reactivation of an infection acquired in the past (latent) (Sawert 1996). Acquired immunodeficiency syndrome (AIDS) is a major risk factor both for reactivation of latent $M$. tuberculosis infection, or primary disease. In areas with low incidence of TB, such as the United States (US), recent data suggest that among foreign-born persons, TB is largely caused by reactivation of latent infection, whereas among US-born persons, a large proportion of cases result from recent transmission (Alland et al. 1994, El Sahly et al. 2001). Therefore, strategies for the control and elimination of TB among foreign-born persons at high risk should be directed toward the treatment of latent TB infection. The restriction fragment-length polymorphism analysis prob-

${ }^{+}$Corresponding author. Fax: $+55-19-3288.0541$. E-mail: mdecr@unicamp.br

Received 3 February 2003

Accepted 21 May 2003 ing insertion sequences (IS6110-RFLP) has been a valuable tool to study TB transmission chains (van Soolingen 2001). This method allows for inferences concerning transmission, based on the similarity of strain fingerprints. The standardization of the method also allows for comparison of the DNA profiles or fingerprints created in different laboratories and tracing of possible transfer of isolates between geographic areas (van Embden et al. 1993). Few genotyping studies have been done in Brazil, given the utility of this tool, such studies should be more recommended to complement the classic epidemiological investigation for full comprehension of TB dynamics, among us.

\section{PATIENTS AND METHODS}

Setting - The study was performed at the Hospital das Clínicas, Universidade Estadual de Campinas (HCUnicamp) in Campinas, state of São Paulo, Brazil. The hospital serves an area with an estimated population of 3 million people. During the last decade an average number of 288 new TB cases were reported in the hospital per year. Patients enrolled in the study were seen at the Infectious Diseases and Lung Services, and Emergency Room.

Case definition - Patients reported to the Hospital Disease Surveillance Office from January 1996 through March 1999, and having an AFB smear positive pulmonary TB were included in the study. The following variables were collected from individual medical records: age, gender, ethnicity, previous TB treatment, diagnosis of 
AIDS or infection by HIV as stated in the patients file, and previous follow-up at the hospital.

M. tuberculosis strains - Isolates were identified as $M$. tuberculosis by positive hybridization with the Accuprobe ${ }^{\circledR}$ test (Gen Probe), subcultured on Löwenstein-Jensen slants, and incubated at $36^{\circ} \mathrm{C}$ for 2 to 3 weeks prior to DNA extraction. Mycobacterial DNA was extracted according to a standard protocol (van Embden et al. 1993).

Molecular strain typing - A total of 76 isolates were submitted for IS6110 RFLP analysis. This method was performed by an international standardized protocol described by van Embden et al. (1993). Fingerprint patterns were analyzed using BioImage Whole Band Analysis program, version 3.2 (An Arbor, Michigan). The DNA fingerprint patterns were compared to the M. tuberculosis database of the Houston Tuberculosis Initiative, located at Baylor College of Medicine, Houston, TX, US, using 100\% similarity criteria.

\section{RESULTS}

Morbidity data - During the study period 1037 cases were reported. Pulmonary cases corresponded to $561 \mathrm{pa}-$ tients, and AFB positive rate was $50 \%$. The absolute number of cases reported, according to the inclusion criteria during the study period, and the number of fingerprints profiles are shown in Fig. 1. A representative amount of strains originated from culture positive patients was genotyped (77.6\%). Some strains could not be typed due to the long storage period.

Molecular analysis - Overall, 59 distinct RFLP patterns were found. Individual fingerprints had from 6 to 21 IS6110 copies (median $=10)$. Cluster analysis revealed that $17(22.3 \%)$ patients were identified from 1 of the 7 recognized clusters ( 2 to 6 patients each). Fingerprints were matched to a collection of strains from the Houston Tuberculosis Initiative, Baylor College of Medicine, Houston, TX, 6 isolates had identical genotype profiles previously characterized in the US, as follows: 3 strains matched with a pattern arbitrarily designated " 137 " found in Houston, 1 isolate matched with pattern " $Z$ " found in El Paso and Ciudad de Juarez, and 2 isolates matched with pattern "AZ", found in New York (Fig. 3). The remaining 11 strains were grouped into 3 new "Brazilian" clusters arbitrarily designated as follows: 2 strains with pattern "BE", 6 with pattern "BF" and 2 with pattern "BH". Pattern "AK", isolated from one patient had been previously found during a cross contamination investigation, that occurred in the Mycobacterial Laboratory of the hospital back in 1995. The clustered isolates were also analyzed concerning the point-in-time they were recovered. Fig. 2 shows that some isolates with the same fingerprint could be identified for as long as 5 years, indicating that some strains (clonal spread) were probably responsible for other non-detected cases which kept them "active" in the community during the study period.

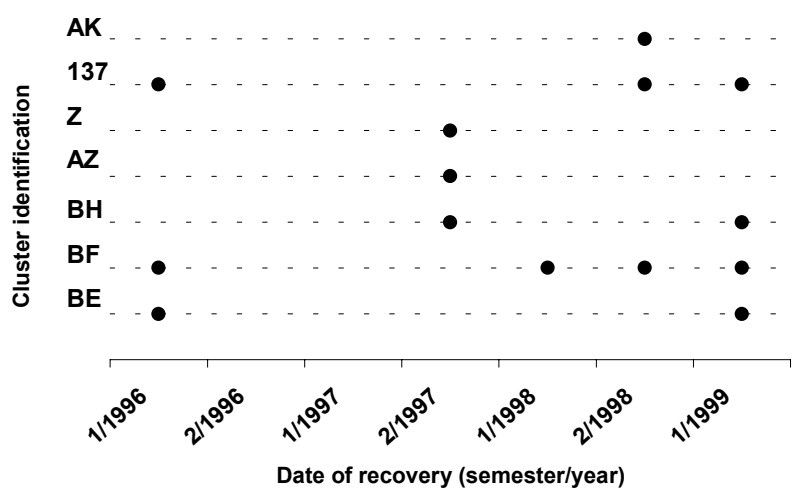

Fig. 2: date of recovery of isolates according to individual clusters.

\section{DISCUSSION}

The advanced understanding of the molecular biology of M. tuberculosis have proven invaluable in unraveling the epidemiology of TB (van Soolingen 2001). The most widespread and reliable genotyping tool is RFLP analysis based on the insertion sequence IS6110. The standardization of this method also allows for comparison of DNA fingerprints between laboratories with the aid of pattern-matching software (van Embden et al. 1993). In the population studied in this work, $22.3 \%$ of all cases

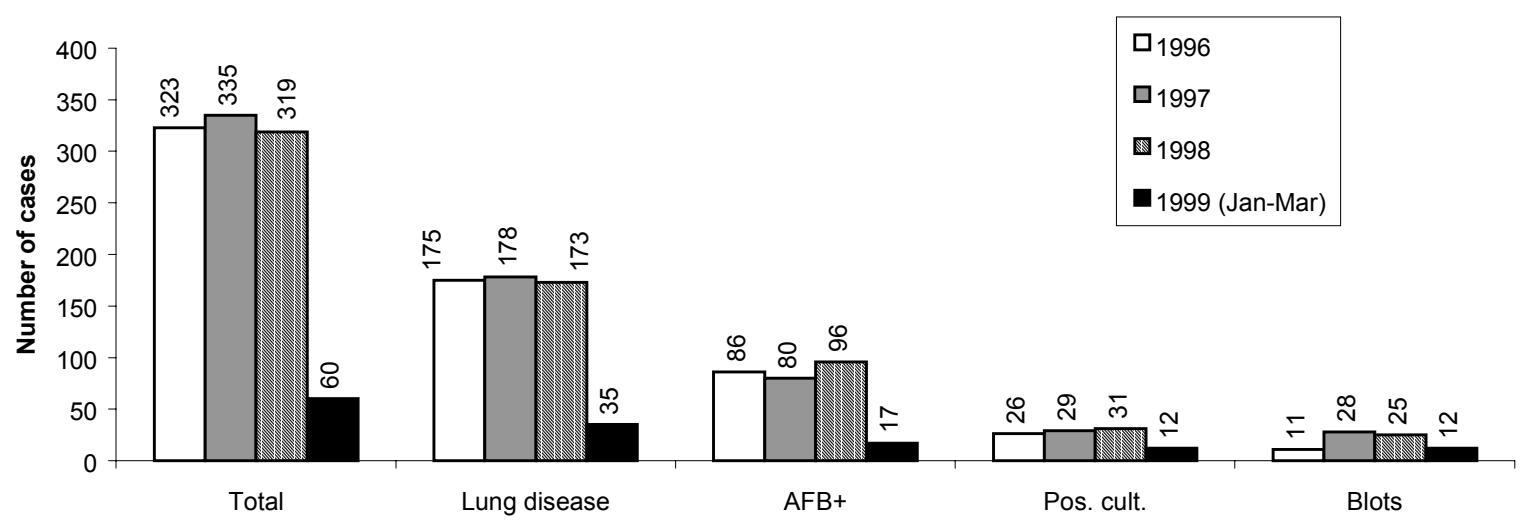

Fig. 1: number of tuberculosis cases reported during the study period. Pulmonary cases, AFB positive patients, culture positive, and absolute number of blots performed are also shown. 


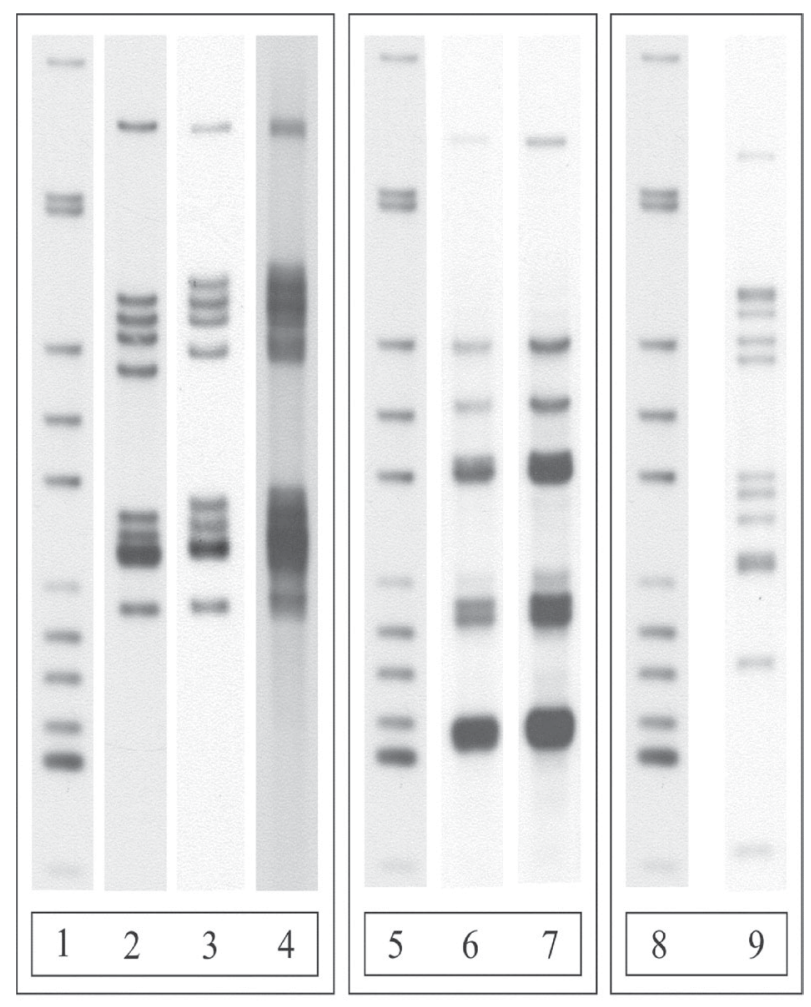

Fig. 3: fingerprints from strains previously isolated in the United States. Lanes - 1, 5, 8: reference strain MT14323; 2, 3, 4: pattern 137 (isolated in Houston); 6, 7 pattern AZ (isolated in New York); 9: pattern Z (isolated in El Paso and Ciudad de Juarez).

were in molecular clusters, suggesting recent transmission of TB. Unfortunately, the low number of strains recovered from the study population and, consequently the low number of genotyped strains, does not allow internal generalization of this result. AFB positive and culture negative/not done patients corresponded to a large amount of cases. In Brazil, molecular typing and population-based data for epidemiology and transmission studies of TB are scarce. Studies performed with patients from reference units for TB and AIDS in metropolitan areas, have shown that $38 \%$ of HIV positive patients attending an outpatient clinic in São Paulo (Ferrazoli et al. 2000), and $19 \%$ of hospitalized, HIV positive patients in Rio de Janeiro (Fandinho et al. 2000) were clustered. Altogether, this data indicate that in developing countries, AIDS associated to TB is often recently acquired. Epidemiologic links among patients infected by clustered strains could not be demonstrated in this work.

Worldwide spread of certain M. tuberculosis strains has recently been documented such as the establishment and subsequent expansion of strains belonging to the Beijing W-cluster genotype (Bifani et al. 1999, 2002). In another study, Suffys et al. (2000) using 219 strains isolated from different patients in Brazil showed that $15 \%$ of the genotype patterns were identical to fingerprints of isolates from a large European database that includes a few South American countries. This data suggests that
TB transmission could be occurring internationally. Interestingly, in our work, in 6 Brazilian cases, M. tuberculosis fingerprints matched with isolates obtained in the US. Even though an extensive investigation was not performed in our work, there were no suspected direct transmission routes between patients infected by clonal strains isolated in both countries. However, those strains were isolated from large metropolitan cities or locations near the US border, which are possible sites of migration and transmission. Together, these studies point to a large, international distribution of $M$. tuberculosis strains. However, the stability of insertion elements, such as IS6110 could be responsible for identical fingerprints, not necessarily reflecting recent transmission. Finally, we strongly recommend a closer technological and data sharing collaboration with public authorities in Brazil and other countries as the US where migration occurs regularly, to describe the true epidemiologic dynamics of tuberculosis.

\section{ACKNOWLEDGMENTS}

To the staff of Mycobacterial Laboratory for initial processing of specimens and Dr Lucilaine Ferrazoli for valuable suggestions.

\section{REFERENCES}

Alland D, Kalkut GE, Moss AR, Mcadam RA, Hahn JA, Bosworth W, Drucker E, Bloom BR 1994. Transmission of tuberculosis in New York City. An analysis by DNA fingerprinting and conventional epidemiologic methods. NEngl J Med 330: 1710-1716.

Bifani PJ, Mathema B, Kurepina NE, Kreiswirth BN 2002. A global dissemination of the Mycobacterium tuberculosis WBeijing family strains. Trends Micorobiol 10: 45-52.

Bifani PJ, Mathema B, Liu Z, Moghazeh SL, Shopsin B, Tempaeski B, Driscol J, Frothingham R, Musser JM, Alcabes P, Kreiswirth BN 1999. Identification of a W variant outbreak of Mycobacterium tuberculosis via population-based molecular epidemiology. JAMA 282:2321-2327.

El Sahly HM, Adams GJ, Soini H, Teeter L, Musser JM, Graviss EA 2001. Epidemiologic differences between United States and foreign-born tuberculosis patients in Houston, Texas. J Infect Dis 183: 461-468.

Fandinho FCO, Kritski AL, Hofer C, Conde Jr H, Ferreira RMC, Saad MHF, Silva MG, Riley LW, Fonseca LS 2000. RFLP patterns and risk factors for recent tuberculosis transmission among hopitalized tuberculosis patients in Rio de Janeiro, Brazil. Trans R Soc Trop Med Hyg 94: 271-275.

Ferrazoli L, Palaci M, Marques LRM, Jamal LF, Afiune JB, Chimara E, Martins MC, Telles MAS, Oliveira CFA, Palhares MC, Spada DTA, Riley LW 2000. Transmission of tuberculosis in an endemic urban setting in Brazil. Int $J$ Tuberc Lung Dis 4: 18-25.

Hopewell PC 1989. Tuberculosis and human immunodeficiency virus infection. Semin Respir Infect 4: 111-122.

Sawert H 1996. The re-emergence of tuberculosis and its economic implications. Pharmacoeconomics 9:379-388.

Soini H, Musser JM 2001. Molecular diagnosis of mycobacteria. Clin Chem 47: 809-814.

Suffys PN, Araújo MEI, Rossetti ML, Zaha A, Barroso EW, Barreto AMW, Campos E, van Soolingen D, Krerner K, Heersma H, Degrave WM 2000 Usefullness of IS6110 restriction fragment length polymorphism typing of Brazilian strains of Mycobacterium tuberculosis and comparison with an international fingerprint database. Res Microbiol 151:343-351. 
van Embden JD, Cave MD, Crawford JT, Dale JW, Eisenach KD, Gicquel B, Hermans P, McAdam R, Shinnick TM, Small PM 1993. Strain identification of Mycobacterium tuberculosis by DNA fingerprinting: recommendations for a standardized methodology. J Clin Microbiol 31: 406-409. van Soolingen D 2001. Molecular epidemiology of tuberculosis and other mycobacterial infections: main methodologies and achievements. J Intern Med 249: 1-26. 